\title{
A facile route to diatoms decorated with gold nanoparticles and their optical properties
}

Alexandra Petzold PhD

Chair for Biogenic Polymers, Campus Straubing for Biotechnology and Sustainability, Technical University of Munich, Straubing, Germany
Cordt Zollfrank PhD

Chair for Biogenic Polymers, Campus Straubing for Biotechnology and

Sustainability, Technical University of Munich, Straubing, Germany

(corresponding author: cordt.zollfrank@tum.de)

Orcid:0000-0002-2717-4161)

This paper presents a facile route for the decoration of biogenic silica from diatoms with gold nanoparticles (AuNPs) with dimensions from 10 to $40 \mathrm{~nm}$. The AuNP decoration on the diatoms is performed through initial attachment of colloidal AuNPs $(2 \mathrm{~nm}$ ) followed by enhancement of the size of the AuNPs by mild reduction of tetrachloroauric (III) acid from solution. The samples were characterised by transmission electron microscopy (TEM), energy-dispersive X-ray analysis, Fourier transform infrared spectroscopy and light spectroscopy in the ultraviolet and visible (UV/Vis) range. The size of the isolated AuNPs on unmodified acid-cleaned diatoms was $10 \mathrm{~nm}$ according to TEM micrograph analysis. The measured UV/Vis spectrum showed an absorption peak at $525 \mathrm{~nm}$, which corresponds well to the optical properties of AuNPs of the same size. The extent of decoration with AuNPs of the diatoms was significantly improved after functionalisation with 3-aminopropyltriethoxysilane of the biogenic silica. The absorption peak in the visible range was redshifted to $590 \mathrm{~nm}$ due to enlargement of the AuNPs up to $40 \mathrm{~nm}$ and clustering of the AuNPs. The AuNP decoration on the hierarchical and complex structured biogenic silica of diatoms might be interesting for biodiagnostic and surface-enhanced Raman spectroscopic applications.

\section{Introduction}

The design and fabrication of complex and hierarchically inorganic architectures with specific functionalities is a rich field of current research. ${ }^{1-4} \mathrm{~A}$ feasible and appealing solution for the problem of generating complex, hierarchically structured materials involves chemical synthesis or deposition of inorganic phases at locally confined sites on predefined material architectures. This is known as the biotemplating approach and defines a general fabrication pathway for complex inorganic materials. ${ }^{4} \mathrm{~A}$ wide variety of complex inorganic materials which cannot be generated by any other method are accessible by biotemplating. ${ }^{1,4}$ Biotemplating involves inorganic phase formation in the presence of a biological structure, which might be basically organic $\left(\right.$ wood $\left.^{5}\right)$, inorganic (diatoms $\left.{ }^{6,7}\right)$ or even a combination of both. ${ }^{8,9}$

The biotemplating approach is of particular interest for the controlled patterning of inorganic nanoparticles. ${ }^{2}$ Noble metal particles ${ }^{10}-$ that is, gold nanoparticles (AuNPs) - are of interest in various applications in biodiagnostics or advanced optical applications such as localised surface plasmon resonance (LSPR) and surface-enhanced Raman spectroscopy $\left(\mathrm{SERS}^{11}\right)$. When a continuous noble metal particle decoration on a surface interacts with light, resonant surface electromagnetic waves result in the absorption of the incident light, which is known as surface plasmon resonance. ${ }^{12}$ Nanoparticulate noble metal materials are of specific interest here because of their strong LSPR. ${ }^{13}$ This electromagnetic potential is derived from the collective oscillation of conduction electrons, called surface plasmons, which were activated by incident light. ${ }^{14}$ This effect is highly sensitive to the size, shape and material composition of the nanostructure. ${ }^{15}$ The material properties of the surrounding medium of the noble metal nanoparticles also determine the resonance frequency of the nanoparticle. The frequency of the LSPR experiences a redshift (lower frequency) with an increase in the dielectric constant of the substrate. ${ }^{16,17}$ The fabrication of a multilayer nanomaterial composed of organic, inorganic and metallic components with interesting optical properties was recently reported. ${ }^{18,19}$ Cellulose nanocrystals were used as a template structure and coated with silica. Gold ( $\mathrm{Au}$ ) and gold-silver nanoparticles from solution were attached to the surface of the cellulose/silica composite by a wet chemical process. By tuning the noble metal particle composition between pure gold and gold-silver, the plasmon resonance could be tuned between 423 and $550 \mathrm{~nm}$. The materials could also be applied as network substrates for SERS. In this context, it would be desirable to use biogenic silica structures directly, which would omit the silica deposition step on a complex structured biotemplate. Biogenic silica is formed by diatoms. ${ }^{20}$ These microalgae are composed of two complex shaped shells (frustules) with hierarchical patterns from the nano- to the micro-scale. The frustules consist of amorphous porous silica with structural features that are difficult to reproduce synthetically. Therefore, diatoms (Coscinodiscus wailesii and Eucampia zodiacus) were used as templates for the decoration with noble metal nanoparticles. ${ }^{21,22}$ Attachment can be achieved through layer-by-layer deposition and covalent linkage. ${ }^{23}$ The fabricated noble metal nanoparticle diatom silica material showed a particular enhancement of SERS intensities. In another approach, the periodic pore pattern of diatom frustules (Cosmioneis hawaiiensis) was exploited for SERS. ${ }^{24}$ AuNPs were self-assembled on the diatom templates and inside the frustule pores. An enhancement in the SERS field on the order of $10^{8}$ was observed. However, a direct correlation of the LSPR and AuNPs on diatom frustules has not been described so far. The present work outlines a simple route for the deposition of AuNPs on diatom 
Bioinspired, Biomimetic and Nanobiomaterials Volume 8 Issue BBN1
A facile route to diatoms decorated with gold nanoparticles and their optical properties

Petzold and Zollfrank frustules (Thalassiosira pseudonana). The optical properties of the AuNP-decorated diatoms were investigated.

\section{Experimental section}

\subsection{Materials and methods}

Suspensions of the acid-cleaned diatoms (T. pseudonana; obtained from the Kröger Group, B Cube - Centre for Molecular Bioengineering, Dresden, Germany) were carefully centrifuged and washed several times with ethanol pro analysi (Roth, Karlsruhe, Germany). Part of the diatom suspension in ethanol was treated with 3-aminopropyltriethoxysilane (APTES, Fluka, Steinheim, Germany) at $90^{\circ} \mathrm{C}$ for $1 \mathrm{~h}$ under reflux and subsequently centrifuged and washed with ethanol pro analysi. The pristine and the APTES-functionalised diatoms were then mixed with a gold sol which was synthesised separately with the reducing agent tetrakis (hydroxymethyl)phosphonium chloride (Sigma-Aldrich, Steinheim, Germany) according to a procedure published by Duff et al. ${ }^{25}$ in 1993. After being stirred for $24 \mathrm{~h}$, the mixture was centrifuged and washed with double-distilled water $\left(\mathrm{H}_{2} \mathrm{O}\right)$ several times until the non-attached nanoparticles were removed from the suspension. In the next step, a gold coating solution composed of tetrachloroauric (III) acid $\left(\mathrm{HAuCl}_{4}\right) /$ potassium carbonate $\left(\mathrm{K}_{2} \mathrm{CO}_{3}\right)(50 \mathrm{ml}$ water, $0.013 \mathrm{~g}$ potassium carbonate, $0.75 \mathrm{ml}$ tetrachloroauric (III) acid (25 mM, Merck, Ismaning, Germany)) was prepared and subsequently reduced by adding $50 \mu \mathrm{l}$ formaldehyde $(37 \mathrm{wt} \%$ in water) (Sigma-Aldrich, Steinheim, Germany). 4-(2-Hydroxyethyl)1-piperazineethanesulfonic acid (1.0 mM Sigma-Aldrich, Steinheim, Germany) was added as a buffer.

\subsection{Characterisation}

For characterisation using a transmission electron microscope (TEM), a drop $(10 \mu 1)$ of the AuNP-decorated diatom suspension in ethanol was placed on a copper grid coated with a $20 \mathrm{~nm}$ carbon film (Plano, Wetzlar, Germany) and dried for $24 \mathrm{~h}$. The TEM investigations were conducted with a Philips CM30 TEM (Eindhoven, the Netherlands) operated at $300 \mathrm{kV}$.

Energy-dispersive X-ray analysis (EDX; Röntec, Germany) was performed on a Zeiss DSM 910A scanning electron microscope (SEM) operated at $10 \mathrm{kV}$.
Optical properties of the AuNP-decorated diatom suspensions in water were measured with an ultraviolet and visible (UV/Vis) spectrometer (J\&M, Tidas, Germany) between 300 and $700 \mathrm{~nm}$.

Infrared spectra of the samples were obtained in attenuated total reflectance geometry with a Fourier transform infrared spectrometer (FTIR; Nicolet 380, Thermo Fisher Scientific, USA) in the wave number region of $4000-500 \mathrm{~cm}^{-1}$.

\section{Results and discussion}

The general scheme for the fabrication of AuNP-decorated NPs is given in Figure 1. First, the diatoms (T. pseudonana) are treated and cleaned with dilute acids to remove organic residues (proteins) and to ensure a hydroxyl group (silanol)-terminated silica surface of the frustule. AuNPs are attached and selfassembled from gold solution either on the acid-cleaned or on the frustules functionalised with APTES.

The TEM investigation of the pristine acid-cleaned diatoms shows a typical spherical appearance with a diameter of $2 \mu \mathrm{m}$. The delicate porous architecture of the diatom including functional elements such as the rimoportulae is clearly visible (Figure 2(a)). The silica of the frustules forms a nanostructured complex network. After treating with the decorating gold solution, AuNPs with sizes between 10 and $20 \mathrm{~nm}$ were found on the silica of the frustules (Figure 2(b)). The observed diameters of the AuNPs correspond well to those of AuNPs found on silica-coated cellulose nanocrystals. ${ }^{18}$ The AuNPs are randomly distributed on the frustule, moderately covering the diatom surface. The presence of gold was confirmed by EDX measurements in the SEM. A closed continuous film is not observed.

However, there seems to be a slight preference for the frustule edge, where several AuNPs are also connected to each other (Figure 2(d)). The attachment of AuNPs to the APTESfunctionalised diatom is obviously very strong as can been seen at a higher magnification in the TEM (Figure 2(e)). The thickness of the silica at the rimoportula walls is only a few nanometres. Even at such low wall thickness, a distinct attachment of AuNP is observed. The selected area electron diffraction shows a wellresolved ring pattern where all relevant rings could be assigned to nanosized and nanocrystalline gold (Figure 2(f)).

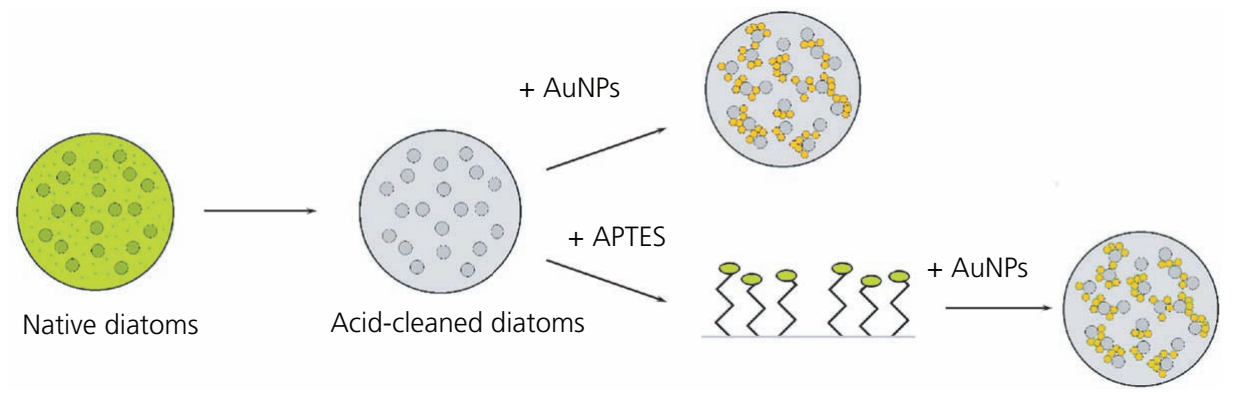

Figure 1. Decoration of acid-cleaned diatoms (T. pseudonana) and of diatoms functionalised with APTES with AuNPs 
Bioinspired, Biomimetic and Nanobiomaterials Volume 8 Issue BBN1
A facile route to diatoms decorated with gold nanoparticles and their optical properties

Petzold and Zollfrank

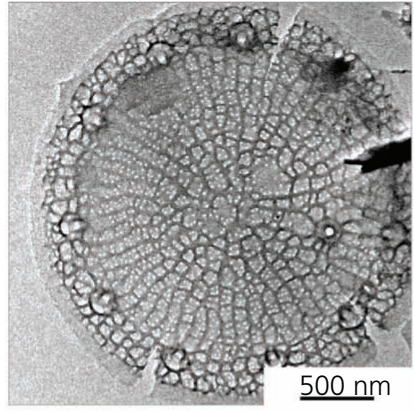

(a)

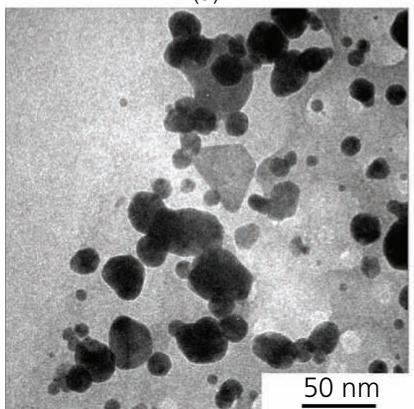

(d)

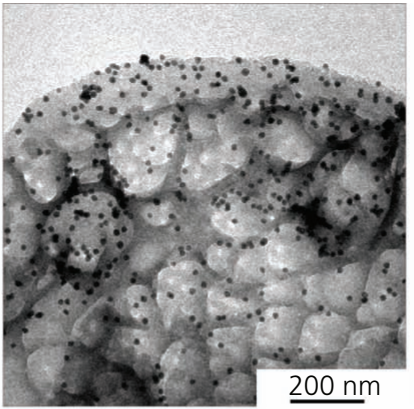

(b)

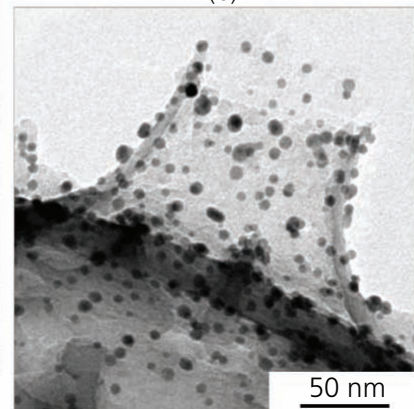

(e)

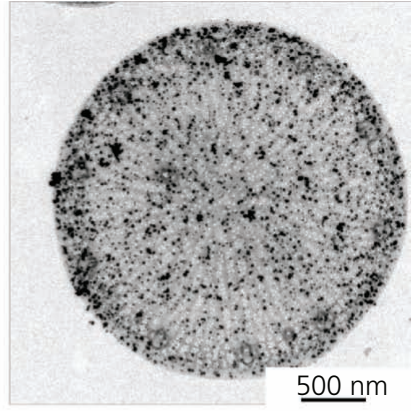

(c)

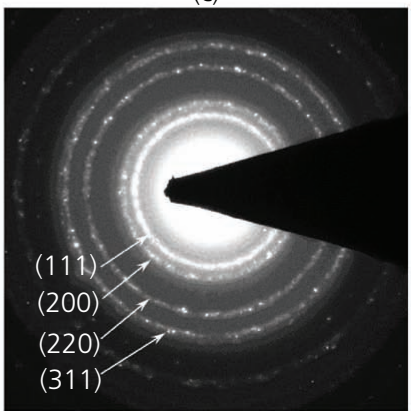

(f)

Figure 2. TEM micrographs of (a) a pristine acid-cleaned diatom (T. pseudonana) and (b) after deposition of AuNPs; (c) an APTESfunctionalised diatom decorated with AuNPs; (d) AuNP clusters on the frustule edge and (e) the decorated nozzle; ( $f$ ) selected area electron diffraction of nanocrystalline AuNPs on the decorated diatom in (c)

The functionalisation with APTES generally improves attachment of the slightly negatively charged AuNPs since the terminal amino function provides a positively charged substrate surface. ${ }^{18}$ The silane groups of APTES covalently bind to the silica surface. The modification of the diatoms with APTES was confirmed by FTIR analysis through the appearance of APTES-derived peaks in the spectra at 1130 and $762 \mathrm{~cm}^{-1}$ (Figure 3).

The freshly prepared reference AuNPs without any templates exhibited a maximum in the UV/Vis absorption spectrum at $520 \mathrm{~nm}$ (Figure 4, a). This value indicates an AuNP size of $10-20 \mathrm{~nm}^{26}$ The observed red colour of the AuNP-modified diatom suspensions was judged based only on a subjective perception. The visual appearance of the AuNPs decorated on APTES-functionalised diatom silica was brownish. As stated earlier, the LSPR strongly depends on the size, shape and dielectric environment of the AuNPs. ${ }^{17,18,26}$ Since the prepared AuNPs from solution and after attachment to the frustule are predominately spherical in appearance, the influence of shape is negligible. The UV/Vis spectrum for AuNP-unmodified diatoms with individual AuNPs shows a peak at $525 \mathrm{~nm}$ (Figure 4, b). This is the same value as observed for the as-prepared AuNPs from solution. This means that the dielectric property of the silica has only a limited effect on the LSPR, when the particle is directly point-connected to the silica surface. ${ }^{17}$ The UV/Vis spectrum for the AuNP decorated on APTES-functionalised diatoms yielded AuNPs with diameters from 10 to $40 \mathrm{~nm}$, which should result in a shift to about $530 \mathrm{~nm} .{ }^{27}$ However, the observed peak for the AuNP-decorated APTES-functionalised diatoms was shifted to $600 \mathrm{~nm}$ (Figure 4, c). This peak shift would correspond to a particle size of around $100 \mathrm{~nm}^{26}$ Such a high shift with a

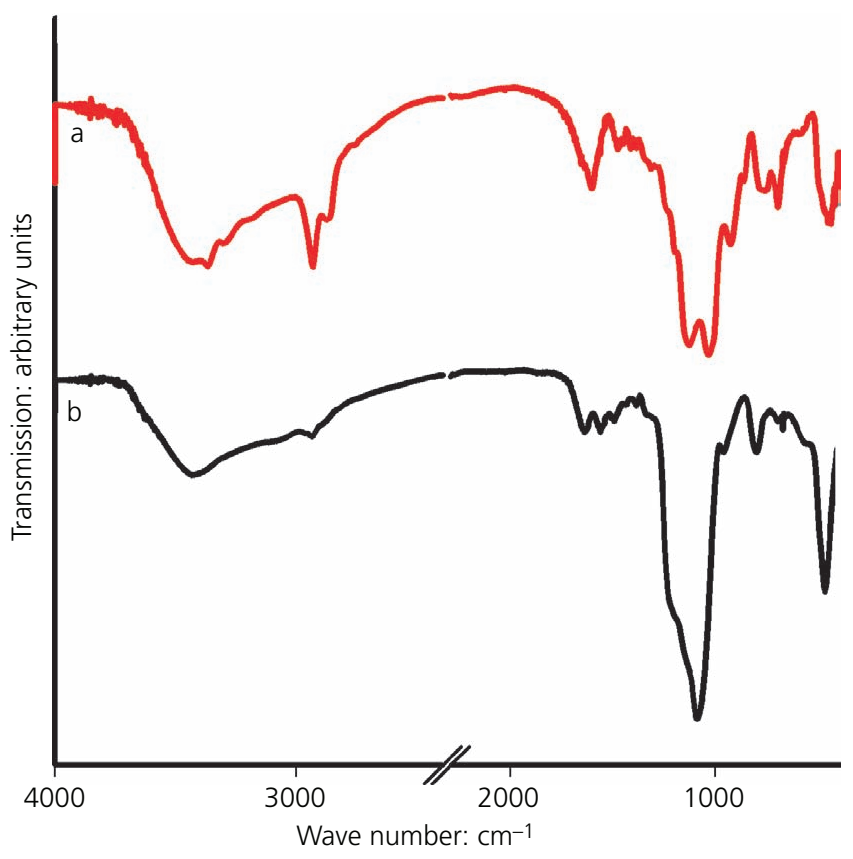

Figure 3. FTIR spectrum of diatoms functionalised with APTES (a) compared to that of pure APTES (b) 
A facile route to diatoms decorated with gold nanoparticles and their optical properties

Petzold and Zollfrank

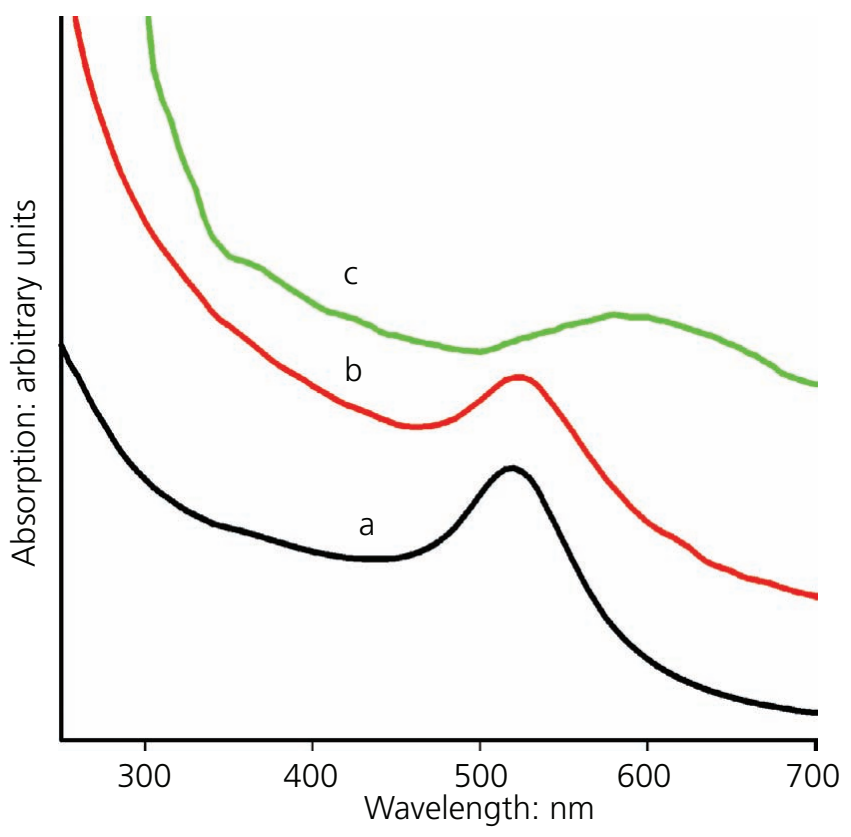

Figure 4. UVNis spectra of as-prepared AuNPS (a), AuNPs decorated on acid-cleaned diatom silica (b) and AuNPs decorated on an APTES-functionalised diatom silica (c)

given particle size can occur only when the particles form interconnected dimers or clusters with a certain aspect ratio. ${ }^{28}$ Since the AuNPs on the APTES-functionalised diatoms partly appear in clusters with dimensions around $50 \mathrm{~nm}$, the observed peak shift is derived from a longitudinal frequency part in the LSPR. $^{26}$ The observed frequency shift in the UV/Vis spectrum is consequently related to the dielectric environment and the shape of the AuNPs in combination with a distinct cluster formation. Furthermore, the peak is extensively broadened, which is due to the AuNP particle size distribution from 10 to $40 \mathrm{~nm}$.

As stated earlier, LSPR is observed on the AuNPs resulting from the collective oscillation of the conduction electrons only when the diameter of the nanoparticle $d_{\mathrm{AuNP}}$ is much smaller than the wavelength of the light $\lambda$ ( $d_{\text {AuNP }} 10-20 \mathrm{~nm} \ll \lambda \approx 520 \mathrm{~nm}$ ). For AuNPs, the resonant excitation of the excitons by the incoming photons at visible wavelengths is responsible for the observed colour. The colour is dependent on the size, solvent, temperature and possibly attached ligands. The dielectric silica of the diatoms, to which the AuNPs are attached, might affect LSPR. $^{29}$ Furthermore, the resonant wavelength can be significantly influenced by the interparticle distance. ${ }^{30}$ As a consequence, the vicinity of other nanoparticles might result in the shift of the LSPR - for example, the aggregation of nanoparticles induces in a significant redshifting from around 520 to around $650 \mathrm{~nm} .^{31,32}$ Using gold nanorods (diameter $10 \mathrm{~nm}$, length $90 \mathrm{~nm}$ ) results in a shift of the LSPR peak to about $1000 \mathrm{~nm}^{33}$ The authors anticipate that the observed redshift is not derived, however, from the attachment of the AuNPs to the nanoparticulate dielectric diatom silica. Coupling between the particles due to the formation of small aggregations is considered to be responsible for the redshift corroborating the authors' earlier findings. ${ }^{18}$

\section{Conclusions}

It could be shown that diatom silica is a suitable template for decoration with AuNPs from solution with a size of $10 \mathrm{~nm}$. The attachment of the AuNPs could be significantly increased by prior functionalisation with APTES. An increased decoration with AuNPs on the biogenic diatom silica was observed, because the silica surface is now positively charged. The particle size distribution was broadened at the same time. The unmodified AuNP-decorated diatoms showed almost no shift of the absorption peak at $525 \mathrm{~nm}$ compared to an AuNP suspension in water with a particle size of $10 \mathrm{~nm}$. The dielectric properties of the biogenic silica of the diatoms exert only a weak to negligible effect on the optical properties of the AuNPs. After functionalisation of the biogenic diatom silica with APTES, decoration with AuNPs was significantly improved. The absorption peak in the visible range was significantly redshifted to $590 \mathrm{~nm}$. This shift could be related to the enlargement of the AuNPs up to a size of $40 \mathrm{~nm}$ size and to some observed clustering of the AuNPs. The observed LSPR from the AuNPs on a hierarchical and complex structured biogenic silica template (diatoms) might be interesting for biodiagnostic and SERS applications.

\section{Acknowledgements}

This work was performed within the frame of Priority Programme SPP 1569 (Generation of multifunctional inorganic materials by molecular bionics) under contract ZO113/17-1 within the first SPP period funded by the German Research Foundation. The assistance of Dr Daniel Van Opdenbosch (Technical University of Munich) with the TEM analysis and valuable discussions is gratefully acknowledged.

\section{REFERENCES}

1. Fan TX, Chow SK and Zhang D (2009) Biomorphic mineralization: from biology to materials. Progress in Materials Science 54(5): 542-659.

2. Zollfrank C, Cromme P, Rauch MWD et al. (2012) Biotemplating of inorganic functional materials from polysaccharides. Bioinspired, Biomimetic and Nanobiomaterials 1(1): 13-25.

3. Meyers MA and Chen PY (2014) Biological Materials ScienceBiological Materials, Bioinspired Materials, and Biomaterials. Cambridge University Press, Cambridge, UK.

4. Zollfrank C, Scheibel T, Seitz H and Travitzky N (2014) Bioinspired materials engineering. In Ullmann's Encyclopedia of Industrial Chemistry (Elvers B (ed.)). Wiley-VCH, Weinheim, Germany, pp. 1-22.

5. Burgert I, Cabane E, Zollfrank C and Berglund L (2015) Bio-inspired functional wood-based materials - hybrids and replicates. International Materials Reviews 60(8): 431-450.

6. Sandhage KH, Dickerson MB, Huseman PM et al. (2002) Novel, bioclastic route to self-assembled, $3 \mathrm{D}$, chemically tailored meso/ nanostructures: shape-preserving reactive conversion of biosilica (diatom) microshells. Advanced Materials 14(6): 429-433.

7. Losic D, Mitchell JG and Voelcker NH (2006) Fabrication of gold nanostructures by templating from porous diatom frustules. New Journal of Chemistry 30(6): 908-914.

8. Scheel H, Zollfrank C and Greil P (2009) Luminescent silica nanotubes and nanowires: preparation from cellulose whisker 
Bioinspired, Biomimetic and Nanobiomaterials Volume 8 Issue BBN1
A facile route to diatoms decorated with gold nanoparticles and their optical properties Petzold and Zollfrank templates and investigation of irradiation-induced luminescence. Journal of Materials Research 24(5): 1709-1715.

9. Gruber S, Gottschlich A, Scheel H et al. (2010) Molecular and supramolecular templating of silica-based nanotubes and introduction of metal nanowires. Physics Status Solidi: Basic Solid State Physics 247(10): 2401-2411.

10. Rosi NL and Mirkin CA (2005) Nanostructures in biodiagnostics. Chemical Reviews 105(4): 1547-1562.

11. Saha K, Agasti SS, Kim C, Li X and Rotello VM (2012) Gold nanoparticles in chemical and biological sensing. Chemical Reviews 112(5): 2739-2779.

12. Tudos AJ and Schasfoort RBM (eds) (2008) Handbook of Surface Plasmon Resonance. RSC Publishing, Cambridge, UK.

13. Oldenburg SJ, Averitt RD, Westcott SL and Halas NJ (1998) Nanoengineering of optical resonances. Chemical Physics Letters 288(2-4): 243-247.

14. Vollmer M and Kreibig U (1995) Optical Properties of Metal Clusters. Springer, Berlin, Germany.

15. Xia Y, Xiong Y, Lim B and Skrabalak SE (2009) Shape-controlled synthesis of metal nanocrystals: simple chemistry meets complex physics? Angewandte Chemie International Edition 48(1): 60-103.

16. Bohren CF and Huffman DR (1998) Absorption and Scattering of Light by Small Particles. Wiley, Weinheim, Germany.

17. Kelly KL, Coronado E, Zhao LL and Schatz GC (2003) The optical properties of metal nanoparticles: the influence of size, shape, and dielectric environment. Journal of Physical Chemistry B 107(3): 668-677.

18. Gruber S, Klupp Taylor RN, Scheel H, Greil P and Zollfrank C (2011) Cellulose-biotemplated silica nanowires coated with a dense gold nanoparticle layer. Materials Chemistry and Physics 129(1-2): 19-22.

19. Gruber S and Zollfrank C (2012) Noble metal nanoparticles on biotemplated nanowires. Bioinspired, Biomimetic and Nanobiomaterials 1(2): 95-100, https://doi.org/10.1680/bbn.11.00010.

20. Round FE, Craford RM and Man DG (1990) The Diatoms. Cambridge University Press, Cambridge, UK

21. Rosi NL, Thaxton CS and Mirkin CA (2004) Control of nanoparticle assembly by using DNA-modified diatom templates. Angewandte Chemie 116(41): 5616-5619.
22. Schröfel A, Kratošová $A$, Bohunická $M$, Dobročka E and Vávra I (2011) Biosynthesis of gold nanoparticles using diatoms-silica-gold and EPS-gold bionanocomposite formation. Journal of Nanoparticle Research 13(8): 3207-3216.

23. Jantschke A, Herrmann AK, Lesnyak V, Eychmüller A and Brunner $E$ (2012) Decoration of diatom biosilica with noble metal and semiconductor nanoparticles $(<10 \mathrm{~nm})$ : assembly, characterization, and applications. Chemistry - an Asian Journal 7(1): 85-90.

24. Chamuah N, Chetia L, Zahan N et al. (2017) A naturally occurring diatom frustule as a SERS substrate for the detection and quantification of chemicals. Journal of Physics D: Applied Physics 50(17): 175103.

25. Duff DP, Baiker A, Gameson I and Edwards PP (1993) A new hydrosol of gold clusters: formation and particle size variation. Langmuir 9(9): 2301-2309.

26. Link S, Wang ZL and El-Sayed MA (1999) Alloy formation of gold-silver nanoparticles and the dependence of the plasmon absorption on their composition. Journal of Physical Chemistry B 103(18): 3529-3533.

27. Stetsenko MO, Rudenko SP, Maksimenko LS et al. (2017) Optical properties of gold nanoparticle assemblies on a glass surface. Nanoscale Research Letters 12: 348-358.

28. Gruber S (2014) Nanopatterning on Cellulose Nanocrystals. PhD thesis, Technical University of Munich, Munich, Germany.

29. Wu C and Xu QH (2009) Stable and functionable mesoporous silicacoated gold nanorods as sensitive localized surface plasmon resonance (LSPR) nanosensors. Langmuir 25(16): 9441-9446.

30. Klar T, Perner M, Grosse S et al. (1998) Surface-plasmon resonances in single metallic nanoparticles. Physical Review Letters 80(19): 4249-4252.

31. Su KH, Wei QH, Zhang X et al. (2003) Interparticle coupling effects on plasmon resonances of nanogold particles. Nano Letters $\mathbf{3 ( 8 )}$ : 1087-1090.

32. Srivastava S, Frankamp BL and Rotello VM (2005) Controlled plasmon resonance of gold nanoparticles self-assembled with PAMAM dendrimers. Chemistry of Materials 17(3): 487-490.

33. Chang $\mathrm{H}$ and Murphy $\mathrm{CJ}$ (2018) Mini gold nanorods with tunable plasmonic peaks beyond $1000 \mathrm{~nm}$. Chemistry of Materials 30(4): $1427-1435$.

\section{How can you contribute?}

To discuss this paper, please submit up to 500 words to the journal office at journals@ice.org.uk. Your contribution will be forwarded to the author(s) for a reply and, if considered appropriate by the editor-in-chief, it will be published as a discussion in a future issue of the journal.

ICE Science journals rely entirely on contributions from the field of materials science and engineering. Information about how to submit your paper online is available at www.icevirtuallibrary.com/page/authors, where you will also find detailed author guidelines. 